\title{
Cooperative Lamb Shift in an Atomic Vapor Layer of Nanometer Thickness
}

\author{
J. Keaveney, ${ }^{1}$ A. Sargsyan, ${ }^{2}$ U. Krohn, ${ }^{1}$ I. G. Hughes, ${ }^{1}$ D. Sarkisyan, ${ }^{2}$ and C. S. Adams ${ }^{1, *}$ \\ ${ }^{1}$ Department of Physics, Rochester Building, Durham University, South Road, Durham DH1 3LE, United Kingdom \\ ${ }^{2}$ Institute for Physical Research, National Academy of Sciences-Ashtarak 2, 0203, Armenia
}

(Received 25 January 2012; published 23 April 2012)

\begin{abstract}
We present an experimental measurement of the cooperative Lamb shift and the Lorentz shift using a nanothickness atomic vapor layer with tunable thickness and atomic density. The cooperative Lamb shift arises due to the exchange of virtual photons between identical atoms. The interference between the forward and backward propagating virtual fields is confirmed by the thickness dependence of the shift, which has a spatial frequency equal to twice that of the optical field. The demonstration of cooperative interactions in an easily scalable system opens the door to a new domain for nonlinear optics.
\end{abstract}

PACS numbers: 42.50.Nn, 32.30.- r, 32.70.Jz

One of the more surprising aspects of quantum electrodynamics is that virtual processes give rise to real phenomena. For example, the Lamb shift [1] arises from a modification of the transition frequency of an atom due to the emission and reabsorption of virtual photons. Similarly, in cavity quantum electrodynamics [2-4] the reflection of the virtual field by a mirror modifies the absorptive and emissive properties of the atom. In a cooperative process such as superradiance, the light-matter interaction is modified by the proximity of identical emitters. The dispersive counterpart of superradiance is known as the cooperative Lamb shift [5] (also sometimes referred to as the collective or $\mathcal{N}$-atom Lamb shift [6]). The cooperative Lamb shift and the cooperative decay rate (i.e., super- or subradiance) arise from the real and imaginary parts of the dipole-dipole interaction, respectively.

Although superradiance has been investigated extensively [7], experimental studies of the cooperative Lamb shift are scarce. Evidence for the shift is restricted to two particular cases, involving three-photon excitation in the limit of the thickness $\ell$ being much larger than the transition wavelength $\lambda$ in an atomic gas [8], and x-ray scattering from Fe layers in a planar cavity [9], demonstrating the fundamental link between the cooperative shift and superradiance. However, the full thickness dependence of the shift in a planar geometry with $\ell<\lambda$ predicted four decades ago [5] has not been observed.

Here we present experimental measurements of the cooperative Lamb shift in a nanothickness vapor layer of $\mathrm{Rb}$ atoms as a function of both density and vapor thickness. The atoms are confined in a cell between two superpolished sapphire plates. Similar nanothickness vapors have been studied extensively over the last two decades, see, e.g., [10-15]. We extend this work to the high density regime where dipole-dipole interactions dominate. In addition, by incorporating the effects of dipole-dipole interactions into a sophisticated model of the absorption spectra we are able to extract the thickness dependence of the resonant shift and thereby verify that the spatial frequency of the cooperative Lamb shift is equal to twice that of the light field [5]. We thus confirm the fundamental mechanism of the cooperative Lamb shift as the exchange of virtual photons.

The underlying mechanism of light scattering is the interference between the incident field and the local field produced by induced oscillatory dipoles. In a medium with $N$ two level dipoles per unit volume, the dipolar field is proportional to the susceptibility which, for a weak field, is given by the steady state solution to the optical Bloch equations (see, e.g., [16]),

$$
\chi=-\frac{N}{\epsilon_{0} \hbar} \frac{d^{2}}{\Delta+i \gamma_{\mathrm{ge}}},
$$

where $d$ is the transition dipole moment, $\gamma_{\mathrm{ge}}$ is the decay rate of the coherence between the ground and excited states, and $\Delta$ is the detuning from resonance. The response of an individual dipole is described in terms of the polarizability,

$$
\alpha_{p}=\frac{\chi}{4 \pi N}=-\frac{1}{4 \pi \epsilon_{0} \hbar} \frac{d^{2}}{\Delta+i \gamma_{\mathrm{ge}}} .
$$

In a dense medium, the field produced by the dipoles modifies the optical response of each individual dipole. This modified response is found by adding the incident field to the dipolar field, $\mathcal{E}_{\text {loc }}=\mathcal{E}+\mathcal{P} / 3 \epsilon_{0}$, where $\mathcal{E}_{\text {loc }}$ is known as the Lorentz local field [17]. The susceptibility determines the bulk response $\mathcal{P}=\epsilon_{0} \chi \mathcal{E}$, whereas the polarizability determines the local response $\mathcal{P}=$ $4 \pi \epsilon_{0} N \alpha_{p} \mathcal{E}_{\text {loc }}$. Substituting for $\mathcal{E}$ and $\mathcal{P}$ we find a relation between the macroscopic variable $\chi$ and the single dipole parameter $\alpha_{p}$ which is referred to as the Lorentz-Lorenz (LL) law [17]:

$$
\chi=\frac{4 \pi N \alpha_{p}}{1-\frac{4}{3} \pi N \alpha_{p}} .
$$

Substituting for $\alpha_{p}$ we find 


$$
\chi=-\frac{N d^{2} / \epsilon_{0} \hbar}{\Delta+i \gamma_{\mathrm{ge}}+N d^{2} / 3 \epsilon_{0} \hbar},
$$

and hence the first order correction due to dipole-dipole interactions is a shift in the resonance frequency known as the Lorentz shift

$$
\Delta_{\mathrm{LL}}=-\frac{N d^{2}}{3 \epsilon_{0} \hbar}
$$

However, as discussed by Stephen [18] and Friedberg, Hartmann, and Manaasah [5], the pairwise dipole-dipole interaction also contains a radiation term. The complete pair potential for two dipoles $V_{\mathrm{dd}}$ has the form

$$
V_{\mathrm{dd}}=\epsilon\left[(1-i k r)\left(3 \cos ^{2} \theta-1\right)+(k r)^{2} \sin ^{2} \theta\right] e^{i k r},
$$

where $\epsilon=-3 \hbar \Gamma / 4(k r)^{3}, r$, and $\theta$ are their separation and relative angle, respectively, and $\Gamma$ is the natural linewidth of the dipole transition with wave vector $k=2 \pi / \lambda$. The real and imaginary parts of $V_{\mathrm{dd}}$ give rise to a level splitting and a modification of the spontaneous lifetime (superradiance or subradiance), respectively [5,18-20]. While these effects have been demonstrated in experiments on two ions [21] and two molecules [22], a key advantage of atomic vapors is that one can easily vary the number of atoms and their mean spacing $\langle r\rangle$. By changing the temperature of the vapor between $20^{\circ} \mathrm{C}$ and $350^{\circ} \mathrm{C}$, one can smoothly vary the number density over 7 orders of magnitude. In doing so, we move between two regimes: $N k^{-3} \ll 1,\langle r\rangle>\lambda$ where dipole-dipole interactions are negligible, and $N k^{-3} \approx 100,\langle r\rangle \sim \lambda / 30$ where dipoledipole interactions dominate.

For more than two dipoles, the cooperative $\mathcal{N}$-atom shift and decay rate are given by a sum of the pairwise dipole-dipole interaction, Eq. (6), for all pairs. For the relatively simple case of an ensemble of dipoles confined within a thin plane of thickness $\ell$, the sum produces a shift [5],

$$
\Delta_{\mathrm{dd}}=-\left|\Delta_{\mathrm{LL}}\right|+\frac{3}{4}\left|\Delta_{\mathrm{LL}}\right|\left(1-\frac{\sin 2 k \ell}{2 k \ell}\right),
$$

where the first term is the Lorentz shift and the second term is the cooperative Lamb shift. There are two remarkable features of Eq. (7). First, the cooperative Lamb shift is a shift to higher energy. One can understand the opposite sign of the Lorentz shift and the cooperative Lamb shift from the pairwise potential, Eq. (6). For a thin slab where all the dipoles lie in the plane, all the dipoles oscillate in phase such that the dipole-dipole interaction reduces to the static case, which, after averaging over all angles, gives an attractive interaction resulting in the Lorentz shift to lower energy. As one moves out of the plane in the propagation direction, the relative phase of the dipoles changes and at a separation of $\lambda / 4$ the second dipole reradiates a field that is $\pi$ out of phase with the source dipole. This switches the sign of the interaction giving rise to the cooperative Lamb shift to higher energies. The second interesting property of the shift is that it depends on twice the propagation phase $k \ell$ which arises due to the reradiation by the second dipole [5]. Finally, we note that while superradiance requires excitation of the medium, the cooperative Lamb shift can be observed in the limit of weak excitation where there is negligible population of the excited state. Consequently, preparation of the medium is not required to observe the shift.

It is important to note that the shift $\Delta_{\mathrm{dd}}$ applies to a static medium. For a gaseous ensemble, atomic motion leads to collisions that also contribute a density dependent shift $\Delta_{\text {col }}$ and broadening $\Gamma_{\text {self }}$ of the resonance lines (see [23] and references therein), and thus the total shift for a thermal ensemble becomes

$$
\Delta_{\mathrm{tot}}=\Delta_{\mathrm{dd}}+\Delta_{\mathrm{col}} .
$$

While evidence for density dependent shifts has been observed in experiments on selective reflection [24], it is important to measure $\Delta_{\text {tot }}$ as a function of the thickness of the medium to separate the thickness independent collisional shift $\Delta_{\text {col }}$ [5] from the thickness dependent cooperative Lamb shift. Below, we present experimental data that allow that distinction to be made for the first time.

To measure the cooperative Lamb shift, we use a gaseous layer of $\mathrm{Rb}$ confined in a vapor cell with thickness $\ell<\lambda$. The cell is shown in Fig. 1(a), and consists of a Rb reservoir and a window region, where the Newton's rings indicate the variation in the cell thickness from $30 \mathrm{~nm}$ at the center to $2 \mu \mathrm{m}$ near the bottom of the photograph. The wedge-shaped thickness profile arises due to the slight curvature of one of the windows (radius of curvature
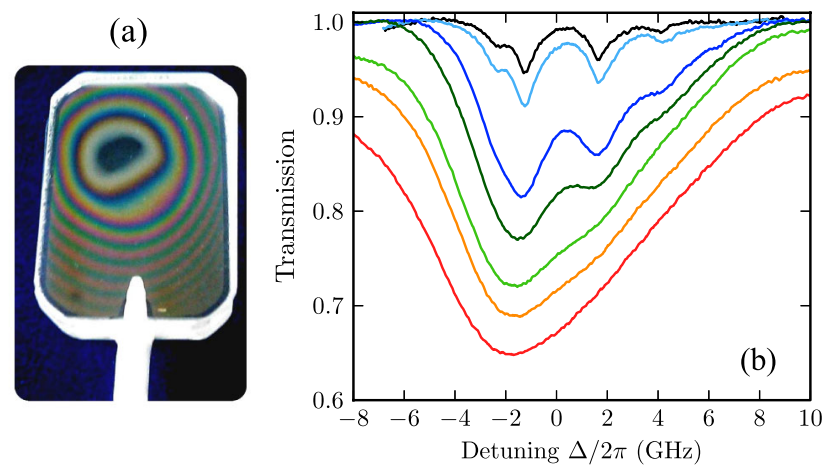

FIG. 1 (color online). Photograph of the nanocell and experimental data. (a) The Newton's rings interference pattern can be observed on the windows of the cell due to the curvature of one of the windows, with a radius of curvature $>100 \mathrm{~m}$. At the center of these rings the cell has its minimum thickness $\ell \sim$ $30 \mathrm{~nm}$, which increases to $\sim 2 \mu \mathrm{m}$ near the stem at the bottom of the photo. (b) Transmission spectra for layer thickness $\ell=$ $90 \mathrm{~nm}$ for measured $\mathrm{Rb}$ temperatures of $190{ }^{\circ} \mathrm{C}$ (black), $207^{\circ} \mathrm{C}$ (light blue), $250{ }^{\circ} \mathrm{C}$ (blue), $265^{\circ} \mathrm{C}$ (dark green), $280{ }^{\circ} \mathrm{C}$ (green), $290^{\circ} \mathrm{C}$ (orange), and $305^{\circ} \mathrm{C}$ (red). The shift can be seen clearly as the density is increased. 
$R>100 \mathrm{~m}$ ). The local thickness at the position of the probe laser is measured at an operational temperature using an interferometric method outlined in Ref. [25]. The local surface roughness measured over an area of $1 \mathrm{~mm}^{2}$ is less than $3 \mathrm{~nm}$, for any part of the window, and the focus of the beam is $\ll 1 \mathrm{~mm}^{2}$. The reservoir can be heated almost independently of the windows and its temperature determines the $\mathrm{Rb}$ number density, while the windows are kept $>50{ }^{\circ} \mathrm{C}$ hotter to prevent condensation of the $\mathrm{Rb}$ vapor. By changing the temperature of the vapor between $20^{\circ} \mathrm{C}$ and $350{ }^{\circ} \mathrm{C}$, we can vary the atomic density between the regimes $N k^{-3} \ll 1$ where dipole-dipole interactions are negligible and $N k^{-3} \approx 100$, where dipole-dipole interactions dominate.

To determine the optical response of the medium, we record transmission spectra as a narrowband laser is scanned across the $D 2$ resonance in $\mathrm{Rb}$ at $780 \mathrm{~nm}$. The light is reduced to a power $P \approx 100 \mathrm{nW}$ and focused to a $30 \mu \mathrm{m}$ spot size inside the cell, leading to a local vapor thickness variation due to the wedge-shaped profile of less than $3 \mathrm{~nm}$. The accuracy in determining the cell thickness is therefore limited by the surface flatness of the windows. Though the intensity of the light is greater than the conventional saturation intensity $\left(I_{\mathrm{sat}} \approx 1.7 \mathrm{~mW} / \mathrm{cm}^{2}\right.$ for the $\mathrm{Rb}$ D2 line), the extremely small thickness of the cell means that optical pumping is strongly suppressed. The transmission is recorded on a photodiode, and a reference cell and Fabry-Perot interferometer are used to calibrate the laser frequency. Example experimental spectra for a thickness of $\ell=90 \mathrm{~nm}$ are shown in Fig. 1(b), where the shift is clearly visible. The shift is extracted by fitting the observed spectra to a comprehensive model of the absolute transmission, based on a Marquardt-Levenberg method (see, e.g., Ref. [26]). The model includes the effect of collisional broadening and has been shown to predict the absolute absorption of the $\mathrm{Rb}$ vapor to better than $0.5 \%$ [23,27]. To this we add the effects of Dicke narrowing [10], where the Doppler effect is partially suppressed as a result of the short length scale, cavity effects [13], since the cell is a low-finesse etalon (with finesse $\mathcal{F} \sim 1$ ), and a single parameter which accounts for a frequency shift of the whole spectrum.

Figure 2 shows experimental data and the theoretical fit for two cases. Panel (a) shows a relatively large vapor thickness $(\ell=\lambda / 2=390 \mathrm{~nm})$ with a low atomic density where dipole-dipole interactions are negligible $\left(\mathrm{Nk}^{-3} \approx\right.$ 0.07), and highlights the effects of Dicke narrowing. Clearly visible are the individual excited state hyperfine components that are normally masked by Doppler broadening in a conventional $\mathrm{cm}$-thickness cell. In stark contrast, panel (b) shows the spectrum obtained in the dipole-dipole dominated regime $\left(N k^{-3} \approx 50\right)$ for a thickness $\ell=$ $90 \mathrm{~nm}$. The individual hyperfine transitions are no longer resolved and there is a clear shift of the resonance to a lower frequency. To illustrate this, we also plot the

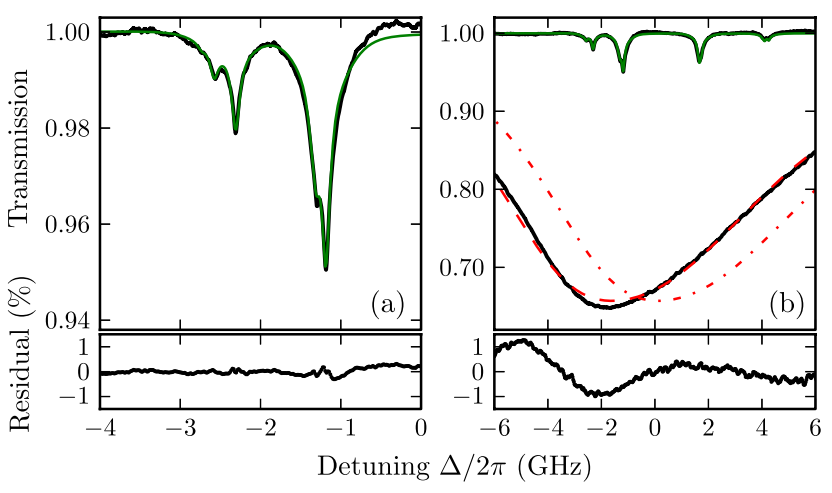

FIG. 2 (color online). Transmission spectra-experiment and theory. Transmission spectrum as a function of linear detuning for thickness (a) $\ell=390 \mathrm{~nm}, N k^{-3} \approx 0.1\left(T=130{ }^{\circ} \mathrm{C}\right)$ and (b) $\ell=90 \mathrm{~nm}, N k^{-3} \approx 50\left(T=305^{\circ} \mathrm{C}\right)$. The black line is experimental data, while the solid green and dashed red lines are the fits to the model outlined in the main text. The dot-dashed red line in panel (b) is the theory without the line shift included. The residuals show the difference between experiment and theory. Zero on the detuning axis represents the weighted line center of the $D 2$ line.

theoretical prediction with the line shift removed. From fitting the data, the collisional broadening is found to be $\Gamma_{\text {self }}=\sqrt{8} \pi N \Gamma k^{-3}=2 \pi\left(1.0 \times 10^{-7}\right) N \mathrm{~Hz} \mathrm{~cm}^{3}$ for thicknesses greater than $\lambda / 4$ in agreement with previous work (see [23] and references therein). For thicknesses shorter than $\lambda / 4$, we observe additional broadening that requires further investigation. We also observe a van der Waals shift due to atom-surface interactions, which we extract by fixing the density and varying the cell thickness (see also [15]); but even for the smallest thickness $(90 \mathrm{~nm})$ this is more than an order of magnitude smaller that the cooperative Lamb shift.

By comparing the experimental data with the theoretical prediction, we extract the line shift as a function of number density and the thickness of the medium. In Fig. 3 we show the measured shift as a function of number density for two thicknesses, $\ell=90$ and $250 \mathrm{~nm}$. Hyperfine splitting gives rise to a different effective dipole for each transition in the spectrum, which at low densities shifts independently. However, in the high density regime $\left(N>10^{16} \mathrm{~cm}^{-3}\right)$ dipole-dipole interactions dominate the line shape and hyperfine splitting becomes negligible. We can then treat the line as a single $s_{1 / 2} \rightarrow p_{3 / 2}$ transition which shifts linearly with the density, as shown in Fig. 3. We fit the gradient of the linear region to obtain the coefficient of the shift, and repeat these measurements for 13 thicknesses up to $600 \mathrm{~nm}$. For thicknesses greater than $600 \mathrm{~nm}$, the high optical depth of the sample impairs the resolution of the line shift. Measurements for a thickness of $420 \mathrm{~nm}$ do not fit to Eq. (8) and have been excluded from the plot. This is thought to be due to the well-known energy pooling process where $420 \mathrm{~nm}$ light is generated by a frequency up conversion of $780 \mathrm{~nm}$ light in a dense Rb vapor [28]. 


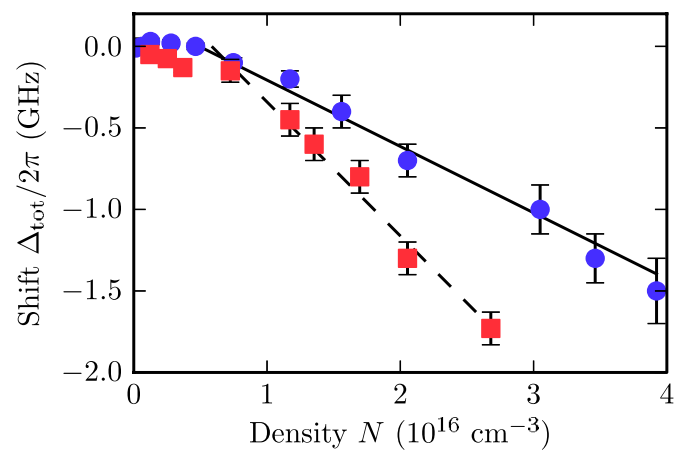

FIG. 3 (color online). Shift of resonance lines with density. Measured shift of resonance lines with density and fit to the linear, high density region for $\ell=90 \mathrm{~nm}$ (red squares, dashed line) and $\ell=250 \mathrm{~nm}$ (blue circles, solid line).

In Fig. 4 we plot the gradient of the line shift as a function of cell thickness. For the Rb D2 resonance, $\Delta_{\mathrm{LL}} / N=-2 \pi \Gamma k^{-3}$, where we have used the relationship between the dipole moment for the $s_{1 / 2} \rightarrow p_{3 / 2}$ transition and the spontaneous decay rate, $d=\sqrt{2 / 3}\left\langle L_{\mathrm{e}}=\right.$ $1 \mid$ er $\left|L_{\mathrm{g}}=0\right\rangle$ (see Ref. [27]). We extract the collisional shift by comparing the data to Eq. (8) with $\Delta_{\text {col }}$ the only free parameter. The amplitude and period of the oscillatory part are fully constrained by Eq. (7). We find the collisional shift to be $\Delta_{\text {col }} / 2 \pi=(-0.25 \pm 0.01) \times 10^{-7} \mathrm{Hzcm}^{3}$, similar to previous measurements on potassium vapor [24]. In this high density limit, the collisional shift is also independent of hyperfine splitting. The solid line is the prediction of Eq. (7), and the agreement between the measured shifts and the theoretical prediction is remarkable (the reduced $\chi^{2}$ for the data is 1.7). As well as measuring the thickness dependence of the cooperative

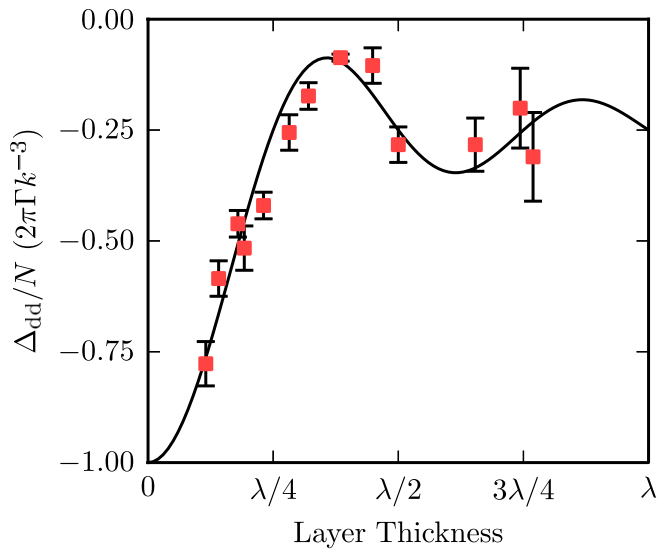

FIG. 4 (color online). Experimental verification of the cooperative Lamb shift. The gradient of the dipole-dipole shift $\Delta_{\mathrm{dd}} / N$ is plotted against cell thickness $\ell$. A collisional shift $\Delta_{\text {col }} / 2 \pi=$ $-0.25 \times 10^{-7} \mathrm{~Hz} \mathrm{~cm}^{3}$ has been subtracted. The solid black line is Eq. (7) with no other free parameters. The conversion between experimental and universal units is outlined in the main text.
Lamb shift, our data also provide a determination of the Lorentz shift which can only be measured in the limit of zero thickness. An important advance on previous studies [8] is that the results clearly show the oscillations in the shift versus the thickness which arises due to the relative phase of the reradiated dipolar field.

The demonstration of the cooperative Lamb shift and coherent dipole-dipole interactions in media with thickness $\sim \lambda / 4$ opens the door to a new domain for quantum optics, analogous to the strong dipole-dipole nonlinearity in blockaded Rydberg systems [29,30]. As the cooperative Lamb shift depends on the degree of excitation [5], exotic nonlinear effects such as mirrorless bistability [31,32] are now accessible experimentally. In addition, given the fundamental link between the cooperative Lamb shift and superradiance, subquarterwave thickness vapors offer an attractive system to study superradiance in the small volume limit. Finally, we note that the measured cooperative Lamb shift is the average dipole-dipole interaction for a homogeneous gas which contains both positive and negative contributions. It could therefore be enhanced by eliminating directions that contribute with the undesired sign, for example, by patterning the distribution of dipoles. These topics will form the focus of future research.

We would like to thank M.P. A. Jones for stimulating discussions. We acknowledge financial support from EPSRC and Durham University.

*c.s.adams@durham.ac.uk

[1] W.E. Lamb and R. C. Retherford, Phys. Rev. 72, 241 (1947).

[2] P. Goy, J. M. Raimond, M. Gross, and S. Haroche, Phys. Rev. Lett. 50, 1903 (1983).

[3] W. Jhe, A. Anderson, E. A. Hinds, D. Meschede, L. Moi, and S. Haroche, Phys. Rev. Lett. 58, 666 (1987).

[4] J. Eschner, C. Raab, F. Schmidt-Kaler, and R. Blatt, Nature (London) 413, 495 (2001).

[5] R. Friedberg, S. R. Hartmann, and J. T. Manassah, Phys. Rep. 7, 101 (1973).

[6] M. O. Scully and A. A. Svidzinsky, Science 328, 1239 (2010).

[7] M. Gross and S. Haroche, Phys. Rep. 93, 301 (1982).

[8] W. R. Garrett, R. C. Hart, J.E. Wray, I. Datskou, and M. G. Payne, Phys. Rev. Lett. 64, 1717 (1990).

[9] R. Röhlsberger, K. Schlage, B. Sahoo, S. Couet, and R. Rüffer, Science 328, 1248 (2010).

[10] S. Briaudeau, S. Saltiel, G. Nienhuis, D. Bloch, and M. Ducloy, Phys. Rev. A 57, R3169 (1998).

[11] D. Sarkisyan, D. Bloch, A. Papoyan, and M. Ducloy, Opt. Commun. 200, 201 (2001).

[12] G. Dutier, A. Yarovitski, S. Saltiel, A. Papoyan, D. Sarkisyan, D. Bloch, and M. Ducloy, Europhys. Lett. 63, 35 (2003).

[13] G. Dutier, S. Saltiel, D. Bloch, and M. Ducloy, J. Opt. Soc. Am. B 20, 793 (2003). 
[14] D. Sarkisyan, T. Varzhapetyan, A. Sarkisyan, Y. Malakyan, A. Papoyan, A. Lezama, D. Bloch, and M. Ducloy, Phys. Rev. A 69, 065802 (2004).

[15] M. Fichet, G. Dutier, A. Yarovitsky, P. Todorov, I. Hamdi, I. Maurin, S. Saltiel, D. Sarkisyan, M.-P. Gorza, D. Bloch, and M. Ducloy, Europhys. Lett. 77, 54001 (2007).

[16] R. Loudon, Quantum Theory of Light (Oxford University Press, Oxford, 1983).

[17] H. A. Lorentz, The Theory of Electrons (BG Teubner, Leipzig, 1909).

[18] M. J. Stephen, J. Chem. Phys. 40, 669 (1964).

[19] R. H. Lehmberg, Phys. Rev. A 2, 889 (1970).

[20] R. Friedberg and J. T. Manassah, Phys. Rev. A 81, 043845 (2010).

[21] R. G. DeVoe and R. G. Brewer, Phys. Rev. Lett. 76, 2049 (1996).

[22] C. Hettich, C. Schmitt, J. Zitzmann, S. Kühn, I. Gerhardt, and V. Sandoghdar, Science 298, 385 (2002).

[23] L. Weller, R.J. Bettles, P. Siddons, C.S. Adams, and I. G. Hughes, J. Phys. B 44, 195006 (2011).
[24] J. J. Maki, M. S. Malcuit, J. E. Sipe, and R. W. Boyd, Phys. Rev. Lett. 67, 972 (1991).

[25] E. Jahier, J. Guéna, P. Jacquier, M. Lintz, A. V. Papoyan, and M. A. Bouchiat, Appl. Phys. B 71, 561 (2000).

[26] I. G. Hughes and T.P. A. Hase, Measurements and Their Uncertainties: A Practical Guide to Modern Error Analysis (Oxford University Press, Oxford, 2010).

[27] P. Siddons, C. S. Adams, C. Ge, and I. G. Hughes, J. Phys. B 41, 155004 (2008).

[28] L. Barbier and M. Cheret, J. Phys. B 16, 3213 (1983).

[29] M. D. Lukin, M. Fleischhauer, R. Cote, L. M. Duan, D. Jaksch, J. I. Cirac, and P. Zoller, Phys. Rev. Lett. 87, 037901 (2001).

[30] J. D. Pritchard, D. Maxwell, A. Gauguet, K. J. Weatherill, M. P. A. Jones, and C. S. Adams, Phys. Rev. Lett. 105, 193603 (2010).

[31] M. Fleischhauer and S. F. Yelin, Phys. Rev. A 59, 2427 (1999).

[32] C. M. Bowden and C.C. Sung, Phys. Rev. A 19, 2392 (1979). 